\title{
Protective effect of restrictive resuscitation on vascular endothelial glycocalyx in pigs with traumatic hemorrhagic shock
}

\author{
Yunfei Chi", Xue Jiang", Jiake Chai, Yang Chang, Tian Liu, Xiangyu Liu, Jianxiang Huang, Bin Wei, \\ Jinguang Zheng, Xingxia Hao, Hailiang Bai, Yirui Qu, Fangchao Hu, Shaofang Han, Qiushuang Wang \\ Burn Institute, The Fourth Medical Center of the PLA General Hospital, Beijing, China \\ Contributions: (I) Conception and design: Y Chi, X Jiang, J Chai, Q Wang; (II) Administrative support: Y Chang, T Liu, X Liu; (III) Provision of \\ study materials or patients: J Huang, B Wei, J Zheng; (IV) Collection and assembly of data: X Hao, H Bai, Y Qu, F Hu, S Han; (V) Data analysis and \\ interpretation: Y Chi, X Jiang; (VI) Manuscript writing: All authors; (VII) Final approval of manuscript: All authors. \\ \#These authors contributed equally to this work. \\ Correspondence to: Jiake Chai; Qiushuang Wang. Burn Institute, The Fourth Medical Center of the PLA General Hospital, 51 Fu Cheng Road, \\ Beijing 100048, China. Email: cjk304@126.com; jennywqs@sina.com.
}

Background: Hemorrhagic shock is the leading cause of early traumatic death. Research and discussion on restrictive fluid resuscitation have been ongoing for many years. The purpose of this study was to explore whether restrictive resuscitation can inhibit the shedding of vascular endothelial glycocalyx in the prehospital treatment of traumatic hemorrhagic shock pigs.

Methods: Landrace pigs were randomly divided into a restrictive resuscitation group (restrictive group) and a conventional resuscitation group (conventional group), with 6 pigs in each group. The gunshot caused a rupture of the pig's receding right femoral artery, and the average arterial pressure was $40-45 \mathrm{mmHg}$ stable for 30 minutes, which was defined as a successful shock model. The end point of resuscitation in the restrictive group was a mean arterial pressure (MAP) of 55-60 $\mathrm{mmHg}$ for 30 minutes, and the end point of resuscitation in the conventional group was a MAP of $70-75 \mathrm{mmHg}$ for 30 minutes. The results of arterial blood gas analysis, hemodynamic indicators, endothelial glycocalyx damage and shedding marker Syndecan1 and soluble thrombomodulin (sTM) expression levels were compared between the two groups of experimental pigs after resuscitation.

Results: The two groups of experimental pigs had the same baseline levels before injury in age, body weight, blood loss, cardiac output index, cardiac function index (CFI), extravascular lung water index (ELWI), and pulmonary vascular permeability index (PVPI). The arterial blood gas analysis of the two experimental pigs showed no significant difference in carbon dioxide partial pressure, oxygen partial pressure, blood oxygen saturation, or blood lactic acid after resuscitation. The difference in cardiac output index and CFI at the end of resuscitation between the two groups was not statistically significant; the absolute value and percentage of Syndecan1 level increase in the restrictive resuscitation group were lower than those in the conventional resuscitation group, and the difference was statistically significant.

Conclusions: Compared with full resuscitation in a short period of prehospital treatment, restrictive resuscitation can achieve a similar effect in maintaining tissue oxygen supply and can reduce the loss of vascular endothelial glycocalyx to a certain extent.

Keywords: Restrictive resuscitation; shock; endothelial glycocalyx; trauma; blood loss

Submitted Nov 26, 2021. Accepted for publication Jan 27, 2022.

doi: 10.21037/atm-21-7004

View this article at: https://dx.doi.org/10.21037/atm-21-7004 


\section{Introduction}

Trauma is the leading cause of death for people under 45 in the United States, and it ranks third among the top ten causes of death for all aged people in the United States (1). Among the trauma patients who died, hemorrhagic shock was the leading cause of early trauma death, accounting for $30-40 \%$ of deaths within 24 hours after trauma (2). Although many explorations have been carried out on the treatment of hemorrhagic shock in recent years, the prehospital mortality rate caused by hemorrhagic shock is still high (3). Among the many studies and discussions on treatment strategies for hemorrhagic shock, the research and discussion on restrictive fluid resuscitation or permissive hypotension resuscitation has been ongoing for many years. The concept of permissive hypotension was first proposed a century ago (4). Its main strategy is to use limited resuscitation crystals in early resuscitation for patients with bleeding that cannot completely stop to ensure perfusion of the main organs. Under these circumstances, a lower-thannormal blood pressure is maintained. Analyzed from the mechanical mechanism, the large amount of fluid entering the systemic circulation within a short time of active fluid resuscitation may increase the hydrostatic pressure and fluid shear force, causing secondary damage to the damaged blood vessel and the formed blood clot, which may lead to additional bleeding caused by treatment (5). In addition, a large amount of fluid below body temperature enters the systemic circulation in a short period of time, which may also cause dilutional coagulopathy and hypothermia $(6,7)$, which can lead to the fatal triad of death in hemorrhagic shock, which includes hypothermia, acidosis and coagulopathy, and an increased rate of mortality. Regarding the therapeutic benefits of restrictive fluid resuscitation, in the different retrospective analyses of penetrating sharp instrument injuries, blunt instrument injuries, and accompanying craniocerebral injuries, most of the results demonstrate the benefits of treatment $(8,9)$.

During hemorrhagic shock and resuscitation, the permeability of the vascular endothelium will increase. The essence is that the normal physiological barrier function of the vascular endothelium is destroyed (10). The vascular endothelial barrier is formed and maintained by the vascular wall, and the vascular wall is composed of the endothelial surface layer, endothelial cells, basement membrane and some auxiliary cells, which together constitute a tight and selective barrier $(11,12)$. Damage to the endothelial surface layer may be the first change in microvascular endothelial dysfunction (13). One of the most important components of the endothelial surface layer is the vascular endothelial glycocalyx (14). The vascular endothelial glycocalyx is closely related to the integrity of the vascular endothelial barrier and changes in vascular endothelial permeability, and it participates in many important physiological and pathological processes of the microvasculature by regulating the interaction between blood cells and endothelial cells and preventing the adherence of white blood cells on the endothelium (15). Therefore, in the early treatment of traumatic hemorrhagic shock, the restrictive fluid resuscitation strategy (maintaining the MAP between $55-60 \mathrm{mmHg}$ ) can be used to protect the vascular endothelial glycocalyx, thereby reducing the permeability of the vascular endothelium. In this study, a model of traumatic hemorrhagic shock was established in $70 \mathrm{~kg}$ class Landrace pigs that were close to human body weight, and the effect and mechanism of restrictive crystal resuscitation in the early stage of shock were explored. The specific methods and results are presented below.

We present the following article in accordance with the ARRIVE reporting checklist (available at https://atm. amegroups.com/article/view/10.21037/atm-21-7004/rc).

\section{Methods}

\section{Laboratory animals and equipment}

\section{Experimental animals}

Twelve healthy Landrace pigs were obtained from Beijing Shichuangshiji Small Pig Breeding Base, license number: SCXK (Beijing) 2018-0011, with a monthly age of $4.58 \pm 0.37$ months and a weight of $69.93 \pm 4.06 \mathrm{~kg}$. The animals were bred adaptively for 1 week under the conditions of a consistent temperature of $25 \pm 3{ }^{\circ} \mathrm{C}$ and consistent humidity of $50 \% \pm 5 \%$ in the laboratory of the Fourth Medical Center of the General Hospital of the People's Liberation Army. Before the experiment, the animals were fasted for 12 hours and were water-free for 4 hours. A protocol was prepared before the study without registration. This study was approved by the Medical Ethics Committee of PLA General Hospital (No. IACUC-2019-0026) and the National Laboratory Animal Management Regulations and guidelines for the care and use of animals were followed.

\section{Animal grouping}

Twelve Landrace pigs were randomly divided into a trauma-hemorrhagic shock restrictive resuscitation group (restrictive group, $\mathrm{n}=6$ ) and a conventional resuscitation group (conventional group, $\mathrm{n}=6$ ) according to the random 
number table.

\section{Main instruments and reagents}

Pulse Indicated Continuous Cardiac Output (PICCO) (MAQUET, Heidelberg, Germany); M9 portable color Doppler (Mindray Biomedical Electronics Co., Ltd., Shenzhen, China); full-featured multiwavelength enzyme label instrument (Dynex, VA, USA); portable blood gas analyzer (OPTI Medical, Atlanta, Georgia, United States); AU480 automatic biochemical analyzer (Beckman Coulter Co., USA); porcine multiligand proteoglycan 1 enzymelinked immunosorbent assay kit (OmnimAbs, USA); porcine soluble thrombomodulin protease-linked immunosorbent assay kit (OmnimAbs, USA); lactated Ringer's solution was purchased from Sinopharm Beijing Tianxing Puxin Biomedical Co., Ltd.

\section{Establishment of hemorrhagic shock model of Landrace pigs}

\section{Methods of anesthesia}

Shutai 50 (Vick, Paris, France) and Sumianxin (Jilin Huamu, China) were mixed in a 2:1 ratio. Five milliliters of Shutai 50 contained $125 \mathrm{mg}$ tiletamine and $125 \mathrm{mg}$ zolazepam. The ingredient was xylazine hydrochloride at a concentration of $0.1 \mathrm{~g} / \mathrm{mL}$. The dosage of mixed anesthetic for ear marginal anesthesia of Landrace pigs was $0.1 \mathrm{~mL} / \mathrm{kg}$. After anesthesia, the patients were intubated orally to avoid tongue suffix asphyxiation. Before injury, $0.3 \mathrm{mg} / \mathrm{kg}$ meloxicam (Baoding Yangguang Bencao, China) was given intramuscularly for analgesia.

\section{Establishment of PICCO hemodynamic monitoring system}

After anesthesia, the Landrace pig limbs were fixed on the curved animal board, and the animal was arranged in a supine position. Using M9 portable color ultrasound as a guide, ultrasound exploration of the right external jugular vein, and guided indwelling deep vein double-lumen catheter, ultrasound exploration of the left femoral artery indwelling PICCO Heat dilution of the arterial catheter, ultrasonic exploration of the bladder, cystostomy, and fixation of $16 \#$ urinary catheter in the bladder. Continuous hemodynamic monitoring was performed throughout the experiment, and the thermal dilution method was used to detect the volume index at each interval.

\section{Modeling of femoral artery gunshot wounds}

After all the tubes were placed, the Landrace pig was fixed on the shooting test bench, the left side lying position was adopted, the right lower limb was raised and fixed on the vertical fixing column of the test bench, and the skin on the left femoral artery was clearly exposed. M9 portable ultrasound exploration was conducted by marking of the right artery skin projection point and subcutaneous injection of $5 \mathrm{ml}$ lidocaine (Sihuan Pharmaceutical, China) at the pre-shooting point for local analgesia and anesthesia. Professional shooting personnel used a 92-type $9 \mathrm{~mm}$ caliber pistol to shoot from 1.5 meters with the marked bullet point, which caused a penetrating injury from the pistol in the right femoral artery area of Landrace pigs and ruptured the right femoral artery. Immediately after shooting, a hemostatic dry glue sponge was used to fill the bullet holes, and pressure was applied for 3 minutes to achieve a state of no active bleeding. After the gunshot wound was locally dissected, the proximal femoral artery was found to be broken, the thrombus was intercepted for pathological examination, and the proximal femoral artery was placed and clamped using the blood sampling bag supporting tube. All operations were completed within 30 minutes after the injury (Figure 1). All blood-stained materials and gauze were collected during the operation, and the amount of bleeding was counted.

\section{Modeling of hemorrhagic shock}

Blood was collected into the bag through the tube at the proximal end of the right femoral artery at a speed of approximately $40-50 \mathrm{~mL} / \mathrm{min}$. Bloodletting will be performed within 30 minutes until the average arterial pressure is stable at $40-45 \mathrm{mmHg}$. If the blood pressure rose, the tube could be opened to let pressure down to $40-45 \mathrm{mmHg}$, and if the average arterial pressure dropped below $40 \mathrm{mmHg}$, autologous blood was infused through the jugular vein to bring the average arterial pressure back to $40-45 \mathrm{mmHg}$. The model was considered successful when the blood pressure stabilized between $40-45 \mathrm{mmHg}$ for 30 minutes.

\section{Resuscitation measures and specimen collection}

\section{Recovery measures}

Conventional resuscitation group: the mean arterial pressure (MAP) stabilized between $40-45 \mathrm{mmHg}$ for 30 minutes to start resuscitation treatment and give Ringer lactate resuscitation through the double-lumen tube of the right jugular vein, the rehydration rate was $20 \mathrm{~mL} / \mathrm{min}$, slowly dripping until the MAP was between $70-75 \mathrm{mmHg}$ 

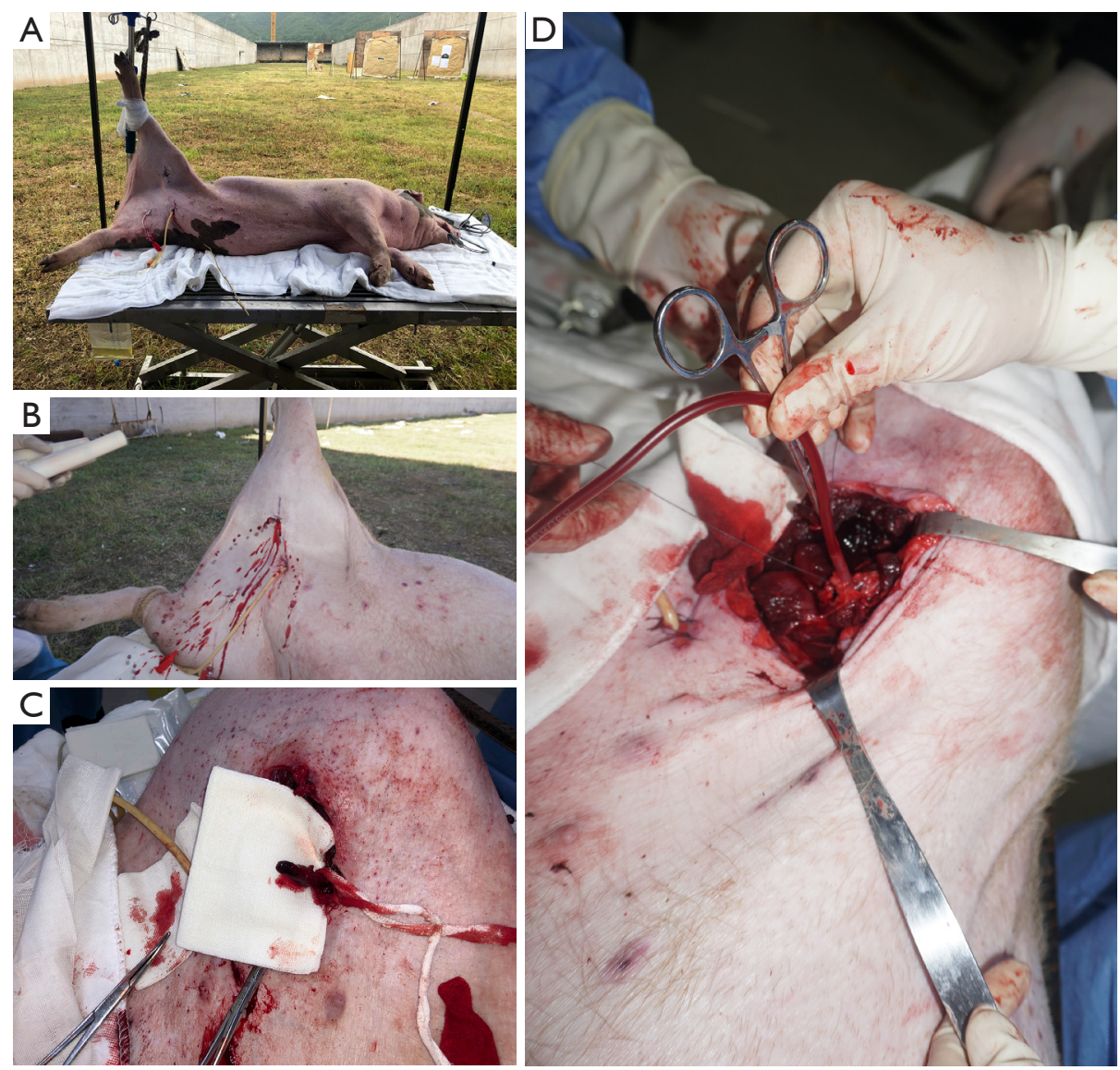

Figure 1 Femoral artery gunshot injury model. (A) Position of the experimental pig before injury was fixed, and the shooting point was located by ultrasound; (B) jet-like hemorrhage at the moment when the femoral artery was ruptured by gunshot; (C) free end of the femoral artery in the local anatomy of the gunshot wound; (D) catheter connected to the blood collection bag at the femoral artery.

and then maintained for 30 minutes, which was regarded as the end point of routine lens resuscitation.

Restrictive resuscitation group: after successful modeling of hemorrhagic shock in Landrace pigs, the MAP stabilized between $40-45 \mathrm{mmHg}$ for 30 minutes to start resuscitation. Resuscitation with lactated Ringer's solution was given through the double-lumen tube of the right jugular vein at a rate of $10 \mathrm{~mL} / \mathrm{min}$ and instilled slowly until the MAP was between $55-60 \mathrm{mmHg}$ and then maintained for $30 \mathrm{~min}$, which was regarded as the end point of restrictive resuscitation.

\section{Serum specimen collection}

Blood samples were collected at various time points, and volume indicators were collected by thermodilution. Blood samples were collected from the femoral artery. The distribution of the time points for the collection of samples was as follows: T0 is before injury after anesthesia, and T1 is after injury and hemostasis (10 minutes after injury), T40 means slow bleeding until the MAP is maintained between $40-45 \mathrm{mmHg}$ for 30 minutes (90 minutes after injury), T55 means the MAP of the restrictive resuscitation group is maintained between $55-60 \mathrm{mmHg}$ for 30 minutes, and the MAP of the T70 conventional resuscitation group between $70-75 \mathrm{mmHg}$ for $30 \mathrm{~min}$. The main detection indicators of blood samples include the main indicators of arterial blood gas analysis, the concentration of Syndecan1, a marker of vascular endothelial glycocalyx damage, and soluble thrombomodulin (sTM), a marker of vascular endothelial cell damage.

\section{Hemodynamic index collection}

PICCO continuous hemodynamic monitoring technology was used to measure the hemodynamic indices of 

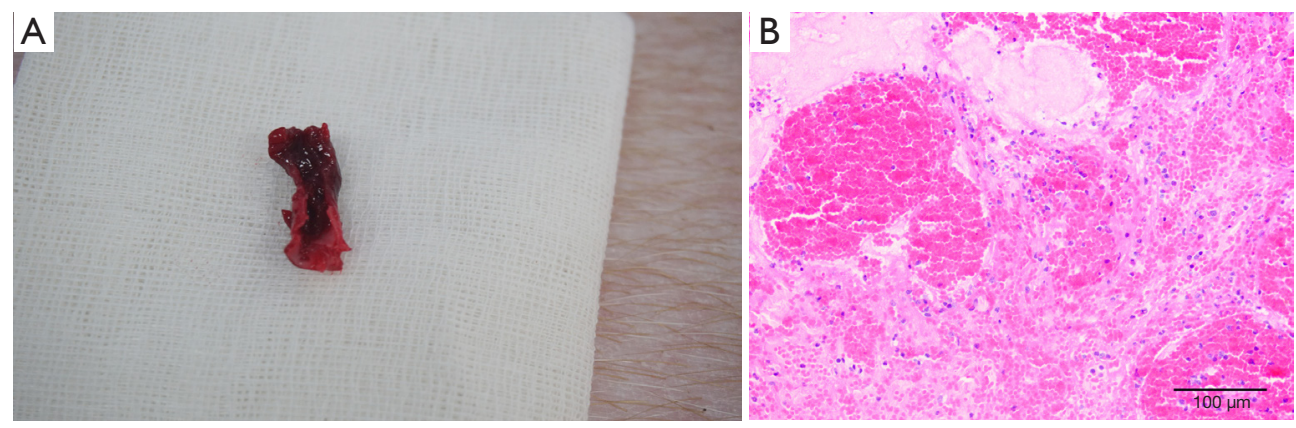

Figure 2 Pathological findings of femoral artery. (A) General anatomy of the femoral artery in a gunshot wound; (B) pathological examination of the stumped vessel by $\mathrm{HE}$ staining $\times 200(\mathrm{bar}=100 \mu \mathrm{m})$. HE, hematoxylin-eosin.

Table 1 Month age, body weight, and hemorrhagic shock bleeding volume of the two groups of Landrace pigs

\begin{tabular}{lccc}
\hline Groups & Age $(\mathrm{m})$ & Weight $(\mathrm{kg})$ & Hemorrhage $(\mathrm{mL})$ \\
\hline Control group $(\mathrm{n}=6)$ & $4.52 \pm 0.39$ & $70.72 \pm 3.14$ & $1,054.17 \pm 189.83$ \\
Observation group $(\mathrm{n}=6)$ & $4.65 \pm 0.37$ & $69.15 \pm 4.99$ & $1,160.00 \pm 370.98$ \\
$\mathrm{P}$ value & 0.5568 & 0.5301 & 0.5478 \\
$\mathrm{t}$ value & 0.6079 & 0.6504 & 0.6221 \\
\hline
\end{tabular}

experimental pigs at various time points, including heart rate (HR), MAP, cardiac index (CI), cardiac function index (CFI), extravascular lung water index (ELWI), and pulmonary vascular permeability index (PVPI).

Detection method: fast ( $<10$ seconds) bolus injection of $15 \mathrm{~mL}$ of low-temperature $0.9 \%$ normal saline $\left(<8{ }^{\circ} \mathrm{C}\right)$ through a deep venous catheter. The saline passes through the superior vena cava-right atrium-right ventriclepulmonary artery-pulmonary vein-left atrium-left ventricleascending. At the receiving end of the aorta-abdominal aorta-femoral artery-PICCO catheter, the computer draws a thermodilution curve for the entire thermodilution process and automatically analyzes the waveform of the curve to obtain relevant parameters. Three measurements were taken continuously at each time point, and the value of each index was averaged.

\section{Statistical analysis}

SPPS22.0 statistical software was used for statistical analysis. Data conforming to a normal distribution are expressed as the mean \pm standard deviation, and the $t$-test was used for comparisons between groups. $\mathrm{P}<0.05$ indicates that the difference is statistically significant.

\section{Results}

\section{Pathological examination}

All experimental pigs were successfully modeled. Local anatomy and pathological examination (hematoxylin-eosin staining) of the ruptured femoral artery in a gunshot wound of an experimental pig are shown in Figure 2.

\section{General information of two groups of Landrace pigs}

The baseline data of the two groups of Landrace pigs (monthly age, body weight, and all blood loss during the successful modeling of a Landrace pig from trauma to hemorrhagic shock) were compared. The $t$-test results showed that the difference between the two groups was not statistically significant, and the baseline level was equivalent. The data are shown in Table 1.

\section{Blood gas analysis at the end of resuscitation in the two groups of Landrace pigs}

The main indicators of arterial blood gas analysis at the end of resuscitation in the two groups of Landrace pigs were compared, including carbon dioxide partial 
Table 2 Arterial gas analysis at the end of resuscitation in the two groups of Landrace pigs

\begin{tabular}{lcccc}
\hline Groups & $\mathrm{PCO}_{2}(\mathrm{mmHg})$ & $\mathrm{PO}_{2}(\mathrm{mmHg})$ & $\mathrm{SO}_{2}(\%)$ & $\mathrm{Lac}(\mathrm{mmol} / \mathrm{L})$ \\
\hline Control group $(\mathrm{n}=6)$ & $46 \pm 8.49$ & $78.83 \pm 4.02$ & $90.83 \pm 0.98$ & $6.65 \pm 2.29$ \\
Observation group $(\mathrm{n}=6)$ & $45 \pm 6.93$ & $72.83 \pm 10.61$ & $89.83 \pm 3.43$ & $5.28 \pm 1.20$ \\
$\mathrm{P}$ value & 0.8276 & 0.2243 & 0.5080 & 0.2217 \\
$\mathrm{t}$ value & 0.2236 & 1.295 & 0.6864 & 1.303 \\
\hline
\end{tabular}

$\mathrm{PCO}_{2}$, partial pressure of carbon dioxide; $\mathrm{PO}_{2}$, partial pressure of oxygen; $\mathrm{SO}_{2}$, oxygen saturation; Lac, lactic acid.

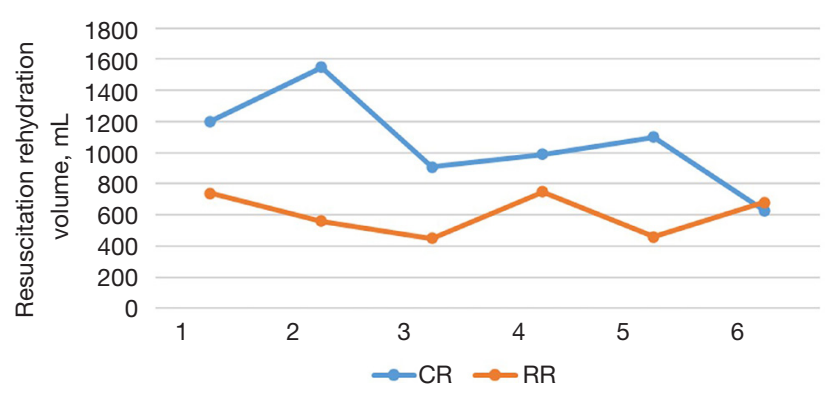

Figure 3 Resuscitation rehydration volume. CR, conventional resuscitation group; RR, restrictive resuscitation group.

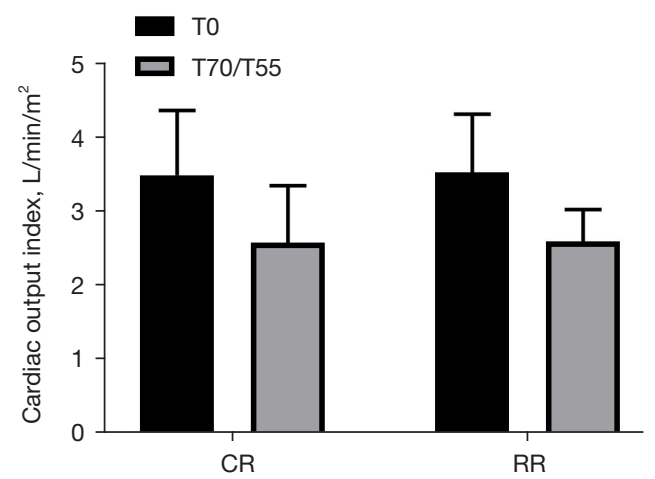

Figure 4 Before and after resuscitation cardiac output index. T0 is before injury after anesthesia, T55 means the MAP of the restrictive resuscitation group is maintained between $55-60 \mathrm{mmHg}$ for 30 minutes, T70 is MAP in conventional resuscitation group between $70-75 \mathrm{mmHg}$ for $30 \mathrm{~min}$. CR, conventional resuscitation group; RR, restrictive resuscitation group; MAP, mean arterial pressure.

pressure $(\mathrm{mmHg})$, oxygen partial pressure $(\mathrm{mmHg})$, blood oxygen saturation (\%), and blood lactic acid ( $\mathrm{mmol} / \mathrm{L})$. The $t$-test showed that there was no statistically significant difference in the main parameters of the blood gas analysis of the Landrace pigs at the end of resuscitation (Table 2).

\section{Amount of fluid needed for resuscitation}

The total amount of liquid resuscitation and supplementation of sodium lactate Ringer's solution in the two groups of Landrace pigs was counted. In the conventional group, the total amount of fluid required for successful fluid resuscitation from traumatic hemorrhagic shock to a stable average arterial pressure of $70-75 \mathrm{mmHg}$ for 30 minutes was $1,063.33 \pm 307.74 \mathrm{~mL}$. In the restrictive group, the total amount of fluid required for successful fluid resuscitation from traumatic hemorrhagic shock modeling to a stable average arterial pressure of $55-60 \mathrm{mmHg}$ for 30 minutes was $606.67 \pm 135.60 \mathrm{~mL}$ (Figure 3).

\section{Hemodynamic changes}

\section{Cardiac output index (CI)}

The CI before injury in the conventional group was $3.50 \pm 0.88 \mathrm{~L} / \mathrm{min} / \mathrm{m}^{2}$, and the CI before injury in the restrictive group was $3.53 \pm 0.79 \mathrm{~L} / \mathrm{min} / \mathrm{m}^{2}(\mathrm{P}=0.9435>0.05)$. The CI after resuscitation in the conventional group was $2.58 \pm 0.77 \mathrm{~L} / \mathrm{min} / \mathrm{m}^{2}$, and the CI after resuscitation in the restrictive group was $2.60 \pm 0.43 \mathrm{~L} / \mathrm{min} / \mathrm{m}^{2}(\mathrm{P}=0.9678>0.05)$. Compared with the preinjury CIs of the two groups of experimental pigs after resuscitation, the post-injury CIs in the two groups both decreased, but the difference was not statistically significant $(\mathrm{P}>0.05)$ (Figure 4).

\section{Comparison of the CFI of the two groups of} experimental pigs before injury and after resuscitation The CFI before injury in the conventional group was $5.85 \pm 1.53 \mathrm{~L} / \mathrm{min}$, and the CFI before injury in the restrictive group was $5.25 \pm 0.90 \mathrm{~L} / \mathrm{min}(\mathrm{P}=0.4281>0.05)$. The CFI after resuscitation in the conventional group was $4.87 \pm 0.54 \mathrm{~L} / \mathrm{min}$, and the CFI after resuscitation in the restrictive group was $4.28 \pm 1.08 \mathrm{~L} / \mathrm{min}(\mathrm{P}=0.2638>0.05)$. Compared with the preinjury heart function index of the two groups of experimental pigs after resuscitation, the CFI 


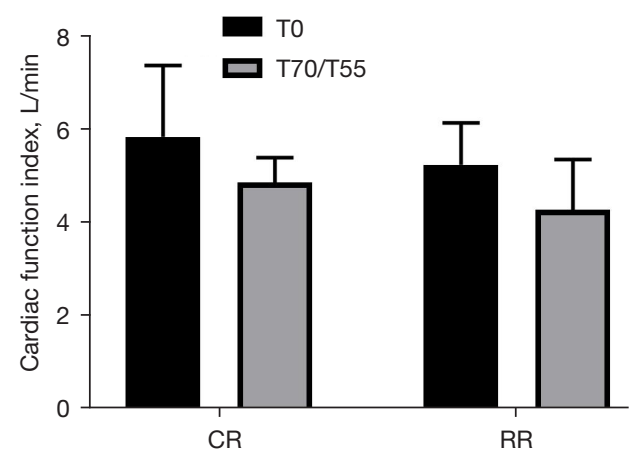

Figure 5 Before and after resuscitation cardiac function index level. T0 is before injury after anesthesia, T55 means the MAP of the restrictive resuscitation group is maintained between 55-60 mmHg for 30 minutes, T70 is MAP in conventional resuscitation group between $70-75 \mathrm{mmHg}$ for $30 \mathrm{~min}$. CR, conventional resuscitation group; RR, restrictive resuscitation group; MAP, mean arterial pressure.

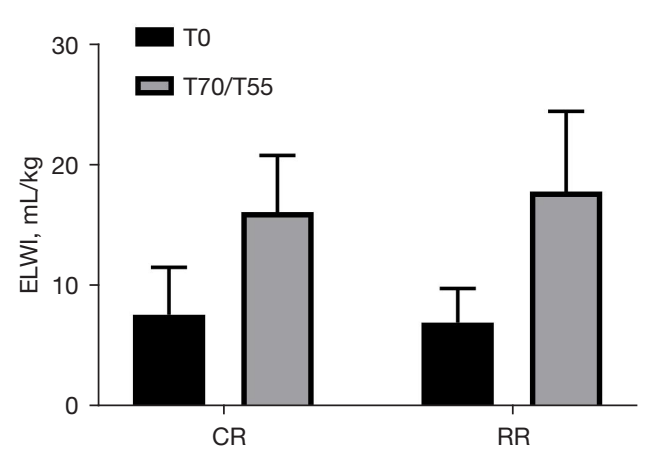

Figure 6 Before and after resuscitation ELWI level. T0 is before injury after anesthesia, T55 means the MAP of the restrictive resuscitation group is maintained between $55-60 \mathrm{mmHg}$ for 30 minutes, T70 is MAP in conventional resuscitation group between $70-75 \mathrm{mmHg}$ for $30 \mathrm{~min}$. ELWI, extravascular lung water index; CR, conventional resuscitation group; RR, restrictive resuscitation group; MAP, mean arterial pressure.

decreased, but the difference was not statistically significant ( $>>0.05)$ (Figure 5).

ELWI of the two groups of experimental pigs compared before injury and after resuscitation

The ELWI before injury in the conventional group was $7.67 \pm 3.93 \mathrm{~mL} / \mathrm{kg}$, and the ELWI before injury in the restrictive group was $7.00 \pm 2.83 \mathrm{~mL} / \mathrm{kg}(\mathrm{P}=0.7430>0.05)$. The ELWI after resuscitation in the conventional group was $16.17 \pm 4.71 \mathrm{~mL} / \mathrm{kg}$, and the ELWI after resuscitation in the

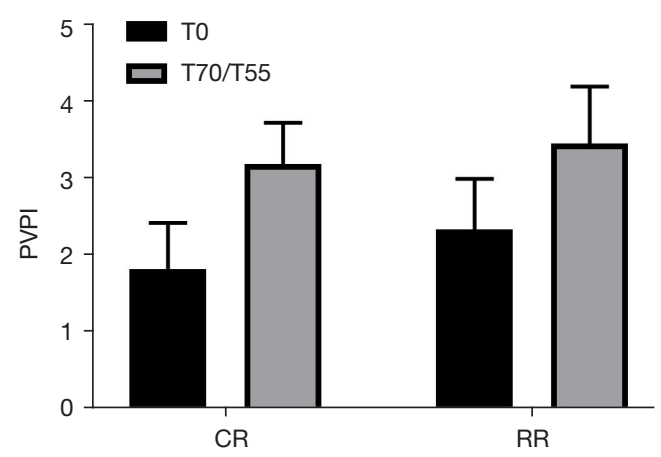

Figure 7 Before and after resuscitation PVPI level. T0 is before injury after anesthesia, T55 means the MAP of the restrictive resuscitation group is maintained between $55-60 \mathrm{mmHg}$ for 30 minutes, T70 is MAP in conventional resuscitation group between $70-75 \mathrm{mmHg}$ for $30 \mathrm{~min}$. PVPI, pulmonary vascular permeability index; CR, conventional resuscitation group; RR, restrictive resuscitation group; MAP, mean arterial pressure.

restrictive group was $17.83 \pm 6.68 \mathrm{~mL} / \mathrm{kg}(\mathrm{P}=0.6281>0.05)$. After resuscitation, the ELWI of the two groups of experimental pigs was significantly higher than that before injury $(\mathrm{P}<0.05$, Figure 6).

PVPI of the two groups of experimental pigs compared before injury and after resuscitation

The PVPI before injury in the conventional group was $1.82 \pm 0.60$, and the PVPI before injury in the restricted group was $2.33 \pm 0.66(\mathrm{P}=0.1865>0.05)$. The PVPI after resuscitation in the routine group was $3.18 \pm 0.54$, and the PVPI after resuscitation in the restricted group was $3.45 \pm 0.74(\mathrm{P}=0.4925>0.05)$. The PVPI of the two groups of experimental pigs after resuscitation was significantly higher than that before injury $(\mathrm{P}<0.05$, Figure 7).

\section{Syndecan1 level changes}

The change in serum Syndecan 1 level at the T55 point of the restrictive group of Landrace pigs (change $=\mathrm{T} 55$ - T0 $=5.13 \pm 1.59 \mathrm{pg} / \mathrm{mL}$ ) vs. the T70 serum Syndecan 1 level at the T70 point of the conventional group of Landrace pigs $($ change $=\mathrm{T} 70-\mathrm{T} 0=8.00 \pm 2.57 \mathrm{pg} / \mathrm{mL}$ ) was compared $(\mathrm{P}=0.0421<0.05$, Figure 8$)$. Comparison of (T55 - T0)/T0 of the conventional group with (T70 T0)/T0 of the restriction group showed that Syndecan1 increased by $39.70 \% \pm 14.05 \%$ in the restrictive group and $70.50 \% \pm 17.38 \%$ in the conventional group $(\mathrm{P}=0.0070<0.05$, Figure 9). 


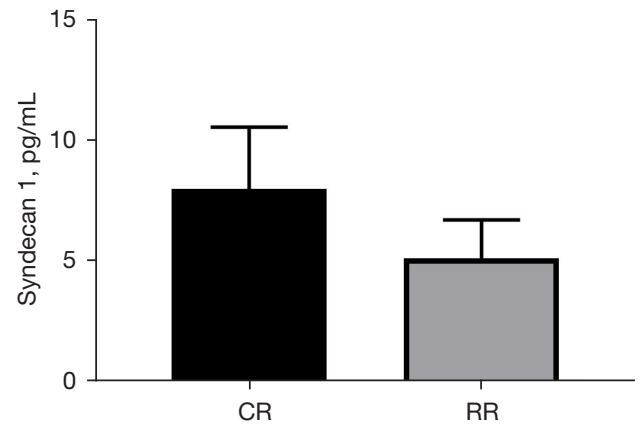

Figure 8 Syndecan 1 increased in CR group. CR, conventional resuscitation group; RR, restrictive resuscitation group.

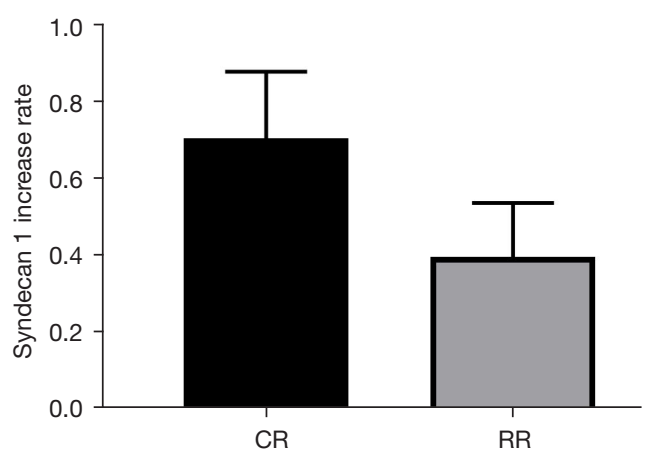

Figure 9 Syndecan 1 increase rate was higher in the conventional resuscitation group than in the restrictive resuscitation group. $\mathrm{CR}$, conventional resuscitation group; RR, restrictive resuscitation group.

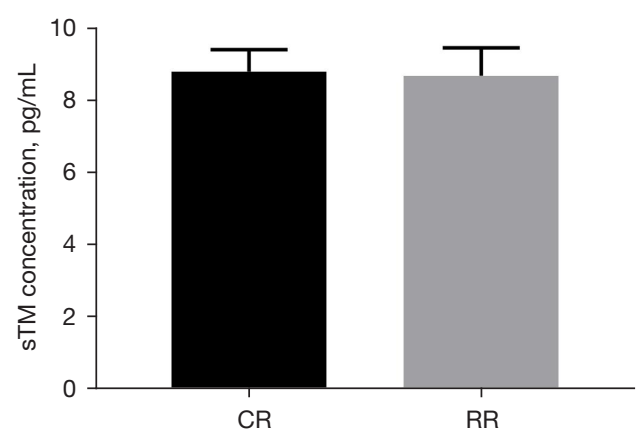

Figure 10 sTM concentration before injury. sTM, soluble thrombomodulin; CR, conventional resuscitation group; RR, restrictive resuscitation group.

\section{Changes of sTM}

The preinjury serum sTM level of the conventional group was $8.80 \pm 0.62 \mathrm{pg} / \mathrm{mL}$, and the preinjury serum sTM level of

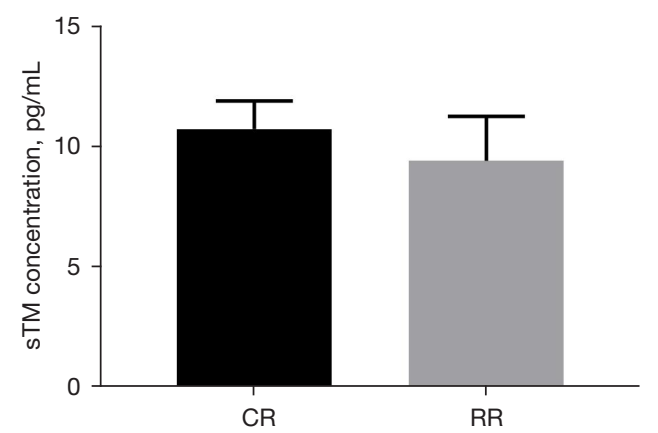

Figure 11 sTM concentration after resuscitation. sTM, soluble thrombomodulin; CR, conventional resuscitation group; RR, restrictive resuscitation group.

the restrictive group was $8.68 \pm 0.78 \mathrm{pg} / \mathrm{mL}(\mathrm{P}=0.7745>0.05$, Figure 10). After resuscitation, the serum sTM level in the conventional group was $10.72 \pm 1.16 \mathrm{pg} / \mathrm{mL}$, and the serum sTM level in the restrictive group was $9.40 \pm 1.85 \mathrm{pg} / \mathrm{mL}$ $(\mathrm{P}=0.1696>0.05$, Figure 11).

\section{Discussion}

In the prehospital treatment stage after severe trauma, the choice of resuscitation method has always been controversial. When using a large amount of fluid for adequate recovery when it is impossible to confirm whether the wound has reached a state of complete hemostasis, the main risks faced may be the shedding of blood clots and rebleeding, dilution coagulopathy and hypothermia (16). However, in most cases, it is relatively difficult to confirm whether the wound has reached a state of complete hemostasis in the prehospital treatment stage. At this time, restrictive resuscitation may involve a smaller risk, which makes restrictive resuscitation accepted in traumatic hemorrhagic shock rescue (17). In recent years, the results of several meta-analyses on the benefits and risks of the two resuscitation methods have also prompted us to conclude that restrictive resuscitation may have more advantages in the prehospital treatment stage of traumatic hemorrhagic shock. Several studies and meta-analyses with survival rate, acute respiratory distress syndrome (ARDS), and multiple organ dysfunction syndrome (MODS) as the main research indicators also suggest that prehospital restrictive resuscitation can improve the survival rate of traumatic hemorrhagic shock and reduce the incidence of ARDS and MODS (18-21). In addition, some researchers have suggested that restrictive resuscitation may lead to an increase in the incidence of acute kidney injury. However, subsequent studies have shown that 
there is no significant difference in the incidence of acute kidney injury between the two resuscitation methods. The researchers analyzed the possible reasons as follows: all patients experienced acute kidney injury of varying degrees during or following trauma (22). Although the controversy and discussion continue, it does not hinder our continuous research on the recovery of restrictive resuscitation.

Previous studies have shown that the occurrence and development of ARDS are closely related to changes in pulmonary vascular endothelial permeability and interstitial edema $(23,24)$, and changes in systemic and pulmonary vascular endothelial permeability during traumatic hemorrhagic shock and resuscitation are also closely related and may be an important factor in the occurrence of ARDS (19). As an important part of the vascular endothelial barrier, the vascular endothelial glycocalyx has been widely recognized for its sentinel role in endothelial barrier destruction and endothelial cell damage (15). Therefore, we consider that if restrictive resuscitation can reduce the incidence of ARDS in patients with traumatic hemorrhagic shock, then we have to wonder whether affecting the permeability of the vascular endothelial barrier is part of the role of protecting the vascular endothelial glycocalyx during resuscitation. We designed this study to explore this question. We randomly divided the two groups of Landrace pigs into a conventional resuscitation group and a restrictive crystal resuscitation group and compared the baseline data of the two experimental groups before injury. Comparison of the two groups of experimental pigs in terms of age, weight, blood loss, CI, CFI, ELWI, and PVPI showed that the abovementioned parameters before injury were not significantly different. Under PICCO continuous hemodynamic monitoring, with different MAPs as the end point of resuscitation, the comparison of some parameters of the two groups of experimental porcine arterial blood gas analysis showed that $\mathrm{PCO}_{2}, \mathrm{PO}_{2}, \mathrm{SO}_{2}$, and lactate were not significantly different after resuscitation. In addition, the CI and CFI at the end of resuscitation between the two groups also showed no significant difference. These results all suggest that restrictive resuscitation can achieve similar effects in maintaining oxygen supply to tissues and organs in a relatively short period of prehospital treatment and that full resuscitation can achieve similar effects on heart function. In the present study, restrictive resuscitation was performed by maintaining the MAP between $55-60 \mathrm{mmHg}$, which was slightly lower than the conventional MAP level in clinical practice.
In this study, to minimize the influence of time factors after shock modeling, we used different bloodletting speeds and fluid supplement speeds to determine the time required for the two groups of experimental pigs to reach the end of resuscitation. After resuscitation, the experimental pig heart output index and heart function index were compared with those before the injury, and the two groups were significantly decreased; the ELWI and PVPI were significantly increased compared with those before the injury. This also truly reflects the progress of the injury. At the end of resuscitation, comparing the serum levels of Syndecan1, a marker of endothelial glycocalyx injury and shedding between the two groups of experimental pigs, the absolute value and percentage increase of Syndecan 1 in the restrictive resuscitation group were lower than those in the conventional resuscitation group, and the difference was statistically significant. This shows to a certain extent that in the early stage of traumatic hemorrhagic shock, restrictive resuscitation can prevent a large amount of solute from entering the circulation in a short period of time and may be able to exert a certain protective effect on the vascular endothelial glycocalyx. However, comparing the levels of sTM, a marker of vascular endothelial cell injury at the end of resuscitation, between the two groups of experimental pigs showed no significant difference. Comparing the ELWI and PVPI at the end of resuscitation between the two groups of experimental pigs, we found no significant difference. Based on these results, we speculate that, due to the limited length of the study, the protective effect of restrictive resuscitation by inhibiting vascular endothelial glycocalyx shedding can be reflected in the results, but whether it can be achieved by reducing vascular endothelial glycocalyx shedding to protect endothelial cells and then protect vascular endothelial barrier function was not confirmed in this study. In addition, there are many factors affecting the barrier function of vascular endothelial cells and the endothelium. With the progression of traumatic hemorrhagic shock and resuscitation, various influencing factors make the final test result more uncertain, which may require further study by increasing the number of animals and extending the study time.

The vascular endothelial glycocalyx is an important part of the vascular endothelial surface layer and participates in many pathophysiological functions related to the vascular endothelium. The glycocalyx is composed of glycoconjugates and proteoglycans. It is not only an important part of the vascular endothelial barrier but also a key element of intercellular and intracellular communication and tissue 
homeostasis (25). In fact, there are many possible factors that affect the endothelial glycocalyx after traumatic hemorrhagic shock, including the secretion of stress hormones, the release of inflammatory factors, changes in fluid mechanics during resuscitation, ischemia-reperfusion injury, and so on. The regulatory mechanism of vascular endothelial glycocalyx is complicated and has not been fully clarified. Based on the research results and literature references, we tried to analyze the reasons why restrictive resuscitation in this study showed a protective effect on endothelial glycocalyx. After successful modeling of traumatic hemorrhagic shock, active resuscitation will cause more crystalloids to enter the systemic circulation in a short time than restrictive resuscitation will, and the entry of a large amount of crystalloids will inevitably lead to an increase in the hydrostatic pressure in the circulation, causing the laminar shear stress to increase and the vascular endothelial glycocalyx to deform (26). Under the influence of many factors after trauma and blood loss, the deformed vascular endothelial glycocalyx may face a greater risk of shedding. In addition, ischemiareperfusion injury during resuscitation from hemorrhagic shock is another important factor affecting the endothelial glycocalyx. A large amount of crystalloid fluid entering the systemic circulation within a short period of time may cause more significant ischemia-reperfusion injury, resulting in capillary post-capillary injury (27). However, a large amount of crystal fluid entering the systemic circulation may cause the rupture of blood clots and dilutional coagulopathy, which may cause further inflammatory factor release and leukocyte adhesion, which are all possible factors that affect the vascular endothelial glycocalyx.

This research is limited by the number of experimental animals, the length of the research, and the collection of indicators. Research related to the mechanism and regulation of the vascular endothelial glycocalyx is a hot spot for current and future research. In short, the results of the study suggest that in the prehospital emergency treatment of traumatic hemorrhagic shock, the application of restrictive resuscitation may exert a certain protective effect on the vascular endothelial glycocalyx compared with the full resuscitation strategy.

\section{Acknowledgments}

Funding: This work was supported by the Major Project of Military Logistical Support Department (No. ALB19J001).

\section{Footnote}

Reporting Checklist: The authors have completed the ARRIVE reporting checklist. Available at https://atm. amegroups.com/article/view/10.21037/atm-21-7004/rc

Data Sharing Statement: Available at https://atm.amegroups. com/article/view/10.21037/atm-21-7004/dss

Conflicts of Interest: All authors have completed the ICMJE uniform disclosure form (available at https://atm.amegroups. com/article/view/10.21037/atm-21-7004/coif). The authors report funding support from the Major Project of Military Logistical Support Department (No. ALB19J001). The authors have no other conflicts of interest to declare.

Ethical Statement: The authors are accountable for all aspects of the work in ensuring that questions related to the accuracy or integrity of any part of the work are appropriately investigated and resolved. This study was approved by the Medical Ethics Committee of PLA General Hospital (No. IACUC-2019-0026) and the National Laboratory Animal Management Regulations and guidelines for the care and use of animals were followed.

Open Access Statement: This is an Open Access article distributed in accordance with the Creative Commons Attribution-NonCommercial-NoDerivs 4.0 International License (CC BY-NC-ND 4.0), which permits the noncommercial replication and distribution of the article with the strict proviso that no changes or edits are made and the original work is properly cited (including links to both the formal publication through the relevant DOI and the license). See: https://creativecommons.org/licenses/by-nc-nd/4.0/.

\section{References}

1. Heron M. Deaths: Leading Causes for 2018. Natl Vital Stat Rep 2021;70:1-115.

2. Cannon JW. Hemorrhagic Shock. N Engl J Med 2018;378:370-9.

3. Duchesne J, Taghavi S, Houghton A, et al. Prehospital Mortality Due to Hemorrhagic Shock Remains High and Unchanged: A Summary of Current Civilian EMS Practices and New Military Changes. Shock 2021;56:3-8.

4. Hardaway RM. Wound shock: a history of its study and treatment by military surgeons. Mil Med 2004;169:265-9. 
5. Torres LN, Sondeen JL, Ji L, et al. Evaluation of resuscitation fluids on endothelial glycocalyx, venular blood flow, and coagulation function after hemorrhagic shock in rats. J Trauma Acute Care Surg 2013;75:759-66.

6. Barak M, Rudin M, Vofsi O, et al. Fluid administration during abdominal surgery influences on coagulation in the postoperative period. Curr Surg 2004;61:459-62.

7. Caminos Eguillor JF, Ferrara G, Kanoore Edul VS, et al. Effects of Systemic Hypothermia on Microcirculation in Conditions of Hemodynamic Stability and in Hemorrhagic Shock. Shock 2021;55:686-92.

8. Tran A, Yates J, Lau A, et al. Permissive hypotension versus conventional resuscitation strategies in adult trauma patients with hemorrhagic shock: A systematic review and meta-analysis of randomized controlled trials. J Trauma Acute Care Surg 2018;84:802-8.

9. M Das J, Anosike K, Waseem M. Permissive Hypotension. StatPearls. Treasure Island (FL), 2021.

10. Klusmeier N, Schnittler HJ, Seebach J. A Novel Microscopic Assay Reveals Heterogeneous Regulation of Local Endothelial Barrier Function. Biophys J 2019;116:1547-59.

11. Alves NG, Motawe ZY, Yuan SY, et al. Endothelial Protrusions in Junctional Integrity and Barrier Function. Curr Top Membr 2018;82:93-140.

12. Vink H, Duling BR. Capillary endothelial surface layer selectively reduces plasma solute distribution volume. Am J Physiol Heart Circ Physiol 2000;278:H285-9.

13. Kurzelewski M, Czarnowska E, Beresewicz A. Superoxideand nitric oxide-derived species mediate endothelial dysfunction, endothelial glycocalyx disruption, and enhanced neutrophil adhesion in the post-ischemic guineapig heart. J Physiol Pharmacol 2005;56:163-78.

14. Van Teeffelen JW, Brands J, Stroes ES, et al. Endothelial glycocalyx: sweet shield of blood vessels. Trends Cardiovasc Med 2007;17:101-5.

15. Pillinger NL, Kam P. Endothelial glycocalyx: basic science and clinical implications. Anaesth Intensive Care 2017;45:295-307.

16. Wang $\mathrm{CH}$, Hsieh WH, Chou $\mathrm{HC}$, et al. Liberal versus

Cite this article as: Chi Y, Jiang X, Chai J, Chang Y, Liu T, Liu X, Huang J, Wei B, Zheng J, Hao X, Bai H, Qu Y, Hu F, Han S, Wang Q. Protective effect of restrictive resuscitation on vascular endothelial glycocalyx in pigs with traumatic hemorrhagic shock. Ann Transl Med 2022;10(4):177. doi: 10.21037/atm-21-7004 restricted fluid resuscitation strategies in trauma patients: a systematic review and meta-analysis of randomized controlled trials and observational studies*. Crit Care Med 2014;42:954-61.

17. Dries DJ. Hypotensive resuscitation. Shock 1996;6:311-6.

18. Owattanapanich N, Chittawatanarat K, Benyakorn T, et al. Risks and benefits of hypotensive resuscitation in patients with traumatic hemorrhagic shock: a meta-analysis. Scand J Trauma Resusc Emerg Med 2018;26:107.

19. Kolarik M, Roberts E. Permissive Hypotension and Trauma: Can Fluid Restriction Reduce the Incidence of ARDS? J Trauma Nurs 2017;24:19-24.

20. Malbrain ML, Marik PE, Witters I, et al. Fluid overload, de-resuscitation, and outcomes in critically ill or injured patients: a systematic review with suggestions for clinical practice. Anaesthesiol Intensive Ther 2014;46:361-80.

21. Rochwerg B, Alhazzani W, Sindi A, et al. Fluid resuscitation in sepsis: a systematic review and network meta-analysis. Ann Intern Med 2014;161:347-55.

22. Ho KH, Tarng YW, Chou YP, et al. Permissive hypotensive resuscitation in patients with traumatic hemorrhagic shock. Scand J Trauma Resusc Emerg Med 2019;27:14.

23. Liu D, Zeng $W$, Zhou $W$, et al. Prognostic value of extra-vascular lung water index and pulmonary vascular permeability index in patients with ARDS. Zhonghua Yi Xue Za Zhi 2015;95:3602-6.

24. Perel A. Extravascular lung water and the pulmonary vascular permeability index may improve the definition of ARDS. Crit Care 2013;17:108.

25. Sieve I, Münster-Kühnel AK, Hilfiker-Kleiner D. Regulation and function of endothelial glycocalyx layer in vascular diseases. Vascul Pharmacol 2018;100:26-33.

26. Weinbaum S, Tarbell JM, Damiano ER. The structure and function of the endothelial glycocalyx layer. Annu Rev Biomed Eng 2007;9:121-67.

27. Platts SH, Linden J, Duling BR. Rapid modification of the glycocalyx caused by ischemia-reperfusion is inhibited by adenosine A2A receptor activation. Am J Physiol Heart Circ Physiol 2003;284:H2360-7. 